\title{
Smartphone Addiction and its Associated Factors Among Freshmen Medical Students in China: A Cross-Sectional Study
}

Huan Liu

Yijishan Hospital of Wannan Medical College

Ming Zhang ( $\nabla$ wnyxyzhangming@foxmail.com )

Wannan Medical College

Zhiqing Zhou

Yijishan Hospital of Wannan Medical College

Ergang Zhu Zhu

Wannan Medical College

Long Huang

Wannan Medical College

\section{Research Article}

Keywords: smartphone addiction, freshmen medical students, China

Posted Date: December 10th, 2021

DOI: https://doi.org/10.21203/rs.3.rs-1071201/v1

License: (c) (i) This work is licensed under a Creative Commons Attribution 4.0 International License. Read Full License 


\section{Abstract}

Background With the widespread smartphone use worldwide, this has brought about addiction and smartphone addiction recognized as an emerging epidemic. The study aims to investigate the prevalence of smartphone addiction among freshmen medical students and explore its association with personal factors, mental health and professional identity.

Methods This cross-sectional study was conducted in October 10th to November 10th, 2020 and included 2182 first-year college students at Wannan Medical College, China.The Smartphone Addiction Test, Professional identity, 12-item General Health Questionnaire were used in this cross-sectional survey. Pearson correlation analysis was performed to examine the correlations between smartphone addiction with mental health and professional identity. Binary logistic regression analysis was conducted to assess examine the factors influencing smartphone addiction.

Results Of the 2182 students, $866(39.7 \%)$ were identified as smartphone addiction. The logistic regression analysis shows that four factors (professional identity scale,poor mental health, whether or not smartphone use before sleep, and perceived study pressure) had a significantly associated with smartphone addiction.

Conclusions This cross-sectional study showed that smartphone addiction was common among the chinese freshmen medical students. smartphone addiction was common among the freshmen medical students surveyed.The findings suggest that the promotion program aimed at enhancing the mental health and professional identity among freshmen medical students are beneficial to reducing their smartphone addiction.

\section{Introduction}

In the last decade, the number of smartphone users has been growing exponentially, and has become an indispensable part of our life.The 45th Statistical Report on China's Internet Development, the current total number of Chinese Internet users was 904 million, nearly $99.3 \%$ of them use their smartphones connect to the Internet as of March 2020[1]. Smartphone is more than just a communication tools, can affording users wide range of features and services, including gaming,shopping,music,viewing videos,online banking, social networking, global positioning system (GPS) navigation, and much more.Although widespread smartphone use have brought many convenience to our lives, the problems caused by overuse of smartphones should not be overlooked.Today, it is apparent that college students have been one of the largest groups of smartphones users, smartphone addiction is a widespread and universal phenomenon among college students.

Smartphone addiction, also referred to as "pathological smartphone use" or "smartphone dependence",which is defined as an individual's uncontrollable his/her use of the smartphone,which could lead to serious harmful activity on work, study, and daily life, it has properties of saliency, impulsivity, highlight behavior, and withdrawal symptoms ${ }^{[2]}$. It is widely acknowledged that smartphone 
addiction not only adversely affects mental health, but also can be detrimental to learning, life and physical health. Researchers have also found that smartphone addiction has been associated with many health outcomes, including fatigue ${ }^{[3]}$, Headache ${ }^{[4]}$, musculoskeletal pain ${ }^{[5]}$, blurred vision ${ }^{[6]}$ and poor sleep quality ${ }^{[7]}$. In addition, for college students, smartphone addiction could seriously affect their academic performance ${ }^{[7]}$,interpersonal competence ${ }^{[8]}$ and emotional problems ${ }^{[9]}$. In light of this,smartphone addic -tion has becomes an urgent emerging public health problem that urgently warrants early prevention and intervention because of its great impact on all aspects of individual's life, study, and social interaction. Although extensive research studies have been conducted on smartphone addiction in the past few years, most studies have focused mainly on college students ${ }^{[10]}$.

When compared to other majors groups, Chinese medical students experience a substantial amount of the long length of schooling, academic pressure, the stress of clinical practice, etc. this is highly likely to cause smartphone addiction ${ }^{[11]}$.Thus, it is important to pay close attention to smartphone addiction in Chinese medical students. In contrast, only a few studies in china that have investigated the prevalence of smartphone addiction among medical students. A cross-sectional study by Chen B et al, the prevalence of smartphone addiction in Chinese medical students was $29.8 \%{ }^{[12]}$. A review of the literature showed that only one study on smartphone addiction among freshmen medical students, nepal newly admitted undergraduate medical students smartphone addiction was $36.8 \%{ }^{[13]}$. In a previous publication, some significantly factors influencing of the smartphone addiction which include gender ${ }^{[12]}$, age ${ }^{[14]}$, monthly income from the family ${ }^{[10]}$, negative emotions ${ }^{[15]}$, high impulsivity and narcissism ${ }^{[16]}$. When starting the present study, few studies have focused on the relationship between smartphone addiction, mental health and professional identity among freshman medical students; this is the focus of the current study.

It has been reported that the medical students in China facing the risk of failing to find a job, due to the expansion of the enrollment scale of the medical colleges, the employment rate of medical graduates has become significantly lower than before,leading to numerous problems such as mental health ${ }^{[17]}$.Prior research has found that people with poor mental health tend to to have greater risk of anxiety and depression ${ }^{[18]}$. Although there are bi-directional associations between smartphone addiction and mental health, early mental health problems may indicate later smartphone addiction. However, the evidence between smartphone addiction and mental health among freshmen in Chinese medical schools was limited. Professional identity among medical students refers to their acceptance and recognition of their chosen major, and their willingness to devote themselves to learning with a positive attitude and positive behavior ${ }^{[19]}$. The medical college is where medical students are likely to experience professional medicine for the first time.Medical students' professional identity is very important because it will influences the school learning and clinical practice. College is a important transition period for student development, professional identity is an important factor influencing success in college. One study by Wang showed a negative correlation between mental health and professional identity in Chinese medical students ${ }^{[20]}$. Therefore, We hypothesized that improved the professional identity of medical students makes an important contribution to improving mental health and smartphone addiction in medical school. 
A high prevalence of smartphone addiction among medical students is a concern because it may affect student behavior, cause learning burnout, and ultimately affect their academic performance and graduation.Therefore, the smartphone addiction of medical students is not only related to the physical and mental health, but also has an important impact on the doctor-patient relationship and the quality of medical care they will provide in the future. Smartphone addiction prevention should pay attention to early detection of the risk factors and intervention for addicted students.In this setting, the current study aimed to examine the prevalence of smartphone addiction in Chinese freshman medical students and explore its association with demographic variables, mental health , and professional identity.Hence, this study main purpose of this cross-sectional study was to investigate the smartphone addiction prevalence and influencing factors of freshmen in a medical school and to provide a reference to reduce the prevalence of smartphone addiction.

\section{Materials And Methods}

\subsection{Participants}

This study used convenient sampling to collect data. This is a network-based cross-sectional study on first-year college students conducted in fall 2020 at a medical school in Anhui Province.The medical students participated voluntarily and anonymously in this study.We collected total of 2222 questionnaires, 40 questionnaires were excluded due to poor filling quality. Finally, our data analysis retained 2182 valid questionnaires for the study, which corresponded to an overall effective response rate was $98.20 \%$. The age of the participants varied from 17 to 22 years, with a mean age of 19.16 years $(S D=1.21)$.

\subsection{Measurement}

\subsubsection{Demographic characteristics}

Based on the relevant literature review, the form includes questions on gender, age, marital status,educational background, years of work experience, professional title,etc.

\subsubsection{Smartphone Addiction}

The degree of smartphone addiction was measured using the Smartphone Addiction Scale-Short Version (SAS-SV), which is a validated scale developed by Kwon in $2013^{[21]}$.It is a self-reported scale consisted of 10 items,each items were rated on a 6 - point Likert scale from 1 to 6 ( $1=$ strongly disagree; $6=$ strongly agree).The total scores were 10-items added together, the overall SAS-SV score ranged from 10 to 60 , and the higher the SAS-SV score indicated the higher level of smartphone addiction.In this study, we adopted a cut-off point of 31 for male and 33 for female to classify smartphone addiction. The Cronbach's alpha in the present study was 0.81 .

\subsubsection{Professional identity}


The Chinese version of professional identity, translated and revised by Qin Panbo ${ }^{[22]}$, was used and included four dimensions:cognition, emotionality, behavior and fitness. The questionnaire consists of 23 items with scores ranged from 23 to 115 based on a five-point Likert scale("complete conformity" $=5$, "conformity" = 4, "neutral" = 3, "inconformity" = 2, and "complete inconformity" =1), the higher the PIQUS score indicating higher the level of professional identity.In this study, Cronbach's alpha was 0.898 .

\subsubsection{The 12-item General Health Questionnaire}

GHQ-12 was used to assess the medical students' mental health ${ }^{[23]}$. The12-item General Health Questionnaire (GHQ-12) is a self-report, 12-item,indicating how often the participants have felt a certain way in the past 2 weeks. All items are answered on a 4-point Likert, which scoring methods 0 (not at all and no more than usual) to 1 (rather more than usual and much more than usual), the final scoring ranges from 0 to 12.Results the higher the score, the more mental health problem,GHQ-12 total score $\geq 3$ was poor mental health.

\subsubsection{Data collection}

Data was collected through an online survey from October 10 th to November 10 th, 2020 . To facilitate the completion of the questionnaires, the questionnaire was performed through the online survey platform using "Questionnaire Star", and the counselors and teachers directly send links to students.All participating medical students need to use the "Questionnaire Star" to submit the questionnaire, and the same IP can only be submitted once.Before issuing the questionnaire, the electronic informed consent form of the medical students was obtained. Participants were told that their participation was entirely voluntary and guaranteed confidentiality,they could terminate the study at any time without reason. The average time needed to complete the questionnaire was approximately $15-25 \mathrm{~min}$.

\subsubsection{Statistical analysis}

In this study, "Questionnaire Star" was used to directly export data in Microsoft Excel format, which was then exported into SPSS20.0 software for statistical analysis.Continuous variables were represented by means \pm standard deviations, categorical variables were represented by frequencies and percentages. The x2-test were occupied to compare differences in characteristics between the smartphone addiction group and the normal groups.Pearson's correlation coefficient ( $r$ ) was used to explore the relationship between smartphone addiction and professional identity. Forward stepwise binary logistic regression analysis was used to determine the predictors of smartphone addiction, and to calculate the odds ratio (OR) and $95 \%$ confidence intervals $(95 \% \mathrm{Cl})$.A two-tailed $\mathrm{P}$ value less than 0.05 was set as statistically significant.

\subsubsection{Ethics}

This study was reviewed and approved by the Ethics Committee of Wan nan Medical College, China.The research protocol and procedures were conducted following the guidelines and principles of the 
Declaration of Helsinki(as revised in 2013).Electronic informed written consent was obtained from all participants prior to the commencement of filling out the questionnaire.

\section{Results}

\subsection{Socio-demographic characteristics of the participants}

A total of 2,182 college students were investigated in this study, of which $620(61.4 \%)$ were males and $696(59.3 \%)$ were females.Age ranged from 17 and 22 years with a mean of $19.65 \pm 0.83$ years.The demographic characteristics of participants (ie, sex,residence,) are described in Table 1.

\subsection{Factors associated with smartphone addiction in the univariate analysis}

Smartphone addiction was detected in 39.7\% (866/2182) based on the smartphone Addiction ScaleShort Version.The results of the $\chi^{2}$ test are presented in Table 1.There were significant differences between residence, perceived Study pressure, professional satisfaction, whether or not Smartphone use before sleep, whether or not student leader and whether or not mental health $(P<0.001)$.No significant relationship was observed between smartphone addiction and gender or whether or not falling in love (all p>.05).

Table 1. Univariate analysis of demographic and mental health characteristics $(\mathrm{N}=\mathbf{2 1 8 2})$. 


\begin{tabular}{|c|c|c|c|c|c|}
\hline \multirow[t]{3}{*}{ variables } & \multicolumn{3}{|c|}{ Smartphone addiction } & \multirow{3}{*}{$x^{2}$} & \multirow[t]{3}{*}{$P$} \\
\hline & No & YES & Total & & \\
\hline & 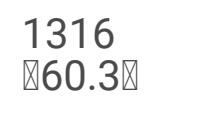 & 866ロ39.70 & $2182 \square 100.0 \square$ & & \\
\hline Gender & & & & 1.011 & 0.315 \\
\hline Male & $\begin{array}{l}620 \\
\square 61.4 \rrbracket\end{array}$ & $\begin{array}{l}389 \\
\varangle 38.6 \rrbracket\end{array}$ & $1009 \rrbracket 46.2 \rrbracket$ & & \\
\hline Female & $\begin{array}{l}696 \\
859.3 \rrbracket\end{array}$ & $\begin{array}{l}477 \\
840.7 \rrbracket\end{array}$ & $1173 \rrbracket 53.8 \rrbracket$ & & \\
\hline Residence & & & & 6.096 & 0.047 \\
\hline Rural area & $\begin{array}{l}728 \\
\square 58.4 \bigotimes\end{array}$ & $\begin{array}{l}519 \\
\square 41.6 \rrbracket\end{array}$ & 1247凶57.1凶 & & \\
\hline Town & $\begin{array}{l}369 \\
\square 61.4 \rrbracket\end{array}$ & $\begin{array}{l}232 \\
\rrbracket 38.6 \rrbracket\end{array}$ & $601 \rrbracket 27.5 \rrbracket$ & & \\
\hline City & $\begin{array}{l}219 \\
\llbracket 65.6 \rrbracket\end{array}$ & $\begin{array}{l}115 \\
\varangle 34.4 \rrbracket\end{array}$ & $334 \rrbracket 15.3 \rrbracket$ & & \\
\hline Perceived Study pressure & & & & 49.437 & $\begin{array}{l}< \\
0.0001\end{array}$ \\
\hline Low & $\begin{array}{l}154 \\
\otimes 71.6 \otimes\end{array}$ & $61 \rrbracket 28.4 \rrbracket$ & $215 \rrbracket 9.9 \rrbracket$ & & \\
\hline Medium & $\begin{array}{l}736 \\
\square 64.8 \square\end{array}$ & $\begin{array}{l}400 \\
\square 35.2 \rrbracket\end{array}$ & $1136 \rrbracket 52.1 \rrbracket$ & & \\
\hline High & $\begin{array}{l}426 \\
₫ 51.3 \rrbracket\end{array}$ & 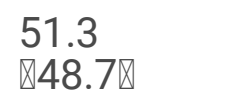 & $831 \rrbracket 38.1 区$ & & \\
\hline Satisfaction with professional & & & & 26.251 & $<$. \\
\hline Low satisfied & $55 \rrbracket 53.9 \rrbracket$ & $47 \rrbracket 46.1 \rrbracket$ & 102ه4.7》 & & \\
\hline Medium satisfied & $\begin{array}{l}610 \\
\square 55.6 \bigotimes\end{array}$ & 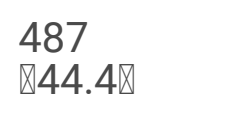 & $1097 \rrbracket 50.3 \rrbracket$ & & \\
\hline Highly satisfied & $\begin{array}{l}651 \\
\rrbracket 66.2 \rrbracket\end{array}$ & $\begin{array}{l}332 \\
\rrbracket 33.8 \rrbracket\end{array}$ & $983 \llbracket 45.1 \rrbracket$ & & \\
\hline $\begin{array}{l}\text { whether or not smartphone use before } \\
\text { sleep }\end{array}$ & & & & 49.115 & $<.0001$ \\
\hline No & $\begin{array}{l}176 \\
₫ 82.6 \rrbracket\end{array}$ & $37 \rrbracket 17.4 \rrbracket$ & $213 \bowtie 9.8 \rrbracket$ & & \\
\hline Yes & 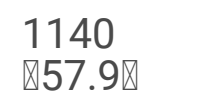 & $\begin{array}{l}829 \\
\nabla 42.1 \rrbracket\end{array}$ & 1969ه90.2》 & & \\
\hline
\end{tabular}




\begin{tabular}{|c|c|c|c|c|c|}
\hline whether or not student leader & & & & 7.047 & 0.008 \\
\hline No & $\begin{array}{l}903 \\
\square 58.5 \rrbracket\end{array}$ & $\begin{array}{l}640 \\
\varangle 41.5 \rrbracket\end{array}$ & 1543ه70.7ه & & \\
\hline Yes & $\begin{array}{l}413 \\
\otimes 64.6 \rrbracket\end{array}$ & $\begin{array}{l}226 \\
\varangle 35.4 \rrbracket\end{array}$ & $639 \llbracket 29.3 \rrbracket$ & & \\
\hline falling in love & & & & 0.245 & 0.621 \\
\hline No & $\begin{array}{l}997 \\
\square 60.6 \rrbracket\end{array}$ & 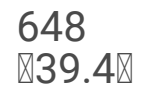 & $1645 \rrbracket 75.4 \rrbracket$ & & \\
\hline Yes & 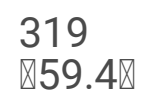 & 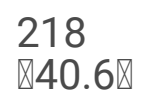 & $537 \rrbracket 24.6 \rrbracket$ & & \\
\hline psychological problems & & & & 43.890 & $\begin{array}{l}<.0001 \\
0\end{array}$ \\
\hline No & 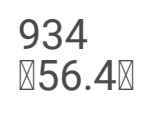 & 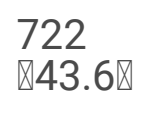 & $526 \rrbracket 24.1 \rrbracket$ & & \\
\hline Yes & $\begin{array}{l}382 \\
\otimes 72.6 \rrbracket\end{array}$ & $\begin{array}{l}144 \\
\otimes 27.4 \rrbracket\end{array}$ & 1656ه75.9凶 & & \\
\hline
\end{tabular}

\subsection{Relationship between Smartphone addiction and Professional Identity of freshmen}

There were statistically significant positive correlations between professional identity scale, cognition scale, emotionality scale, and fitness scores and smartphone addiction scores $(r=0.794, p=0.000 ; r=$ $0.448, p=0.000 ; r=0.566, p=0.000 ; r=0.710, p=0.000$; and $r=0.640, p=0.000$, respectively) (Table 2).

Table 2. Correlation between Smartphone addiction and Professional Identity

\begin{tabular}{|lll|}
\hline variables & \multicolumn{2}{l|}{ Smartphone addiction } \\
\cline { 2 - 3 } & $r$ & $P$ \\
\hline Cognition & $0.448^{\star \star}$ & 0.000 \\
\hline Emotionality & $0.566^{\star \star}$ & 0.000 \\
\hline Behavior & $0.710^{\star *}$ & 0.000 \\
\hline Fitness & $0.640^{\star \star}$ & 0.000 \\
\hline Professional Identity Scale & $0.794 * \star$ & 0.000 \\
\hline
\end{tabular}

\subsection{Factors Associated With Smartphone addiction}

As Risk factors for increases in the severity of smartphone addiction included being Poor mental health $(\mathrm{OR}=1.677,95 \% \mathrm{Cl}=1.338-2.102)$, being smartphone use before sleep $(\mathrm{OR}=3.120,95 \% \mathrm{Cl}=$ 2.147-4.536), perceived study pressure medium $(\mathrm{OR}=1.265,95 \% \mathrm{Cl}=0.908-1.762)$ and perceived study 
pressure High $(\mathrm{OR}=2.005,95 \% \mathrm{Cl}=1.430-2.811)$. Professional Identity $(\mathrm{OR}=0.982,95 \% \mathrm{Cl}=0.975-0.988)$ was a protective factor for smartphone addiction (Table 3 ).

Table 3. Logistic regression analysis for factors associated with Smartphone addiction

\begin{tabular}{|llccccc|}
\hline Variable & B & SE & Wald & $P$ & OR & 95\% Cl \\
\hline Professional Identity Scale & -0.019 & 0.004 & 26.830 & 0.000 & 0.982 & $\begin{array}{c}0.975- \\
0.988\end{array}$ \\
\hline Poor mental health & 0.517 & 0.115 & 20.142 & 0.000 & 1.677 & $\begin{array}{c}1.338- \\
2.102\end{array}$ \\
\hline $\begin{array}{l}\text { whether or not Smartphone use before } \\
\text { sleep }\end{array}$ & 1.138 & 0.191 & 35.551 & 0.000 & 3.120 & $\begin{array}{l}2.147- \\
\text { Perceived Study pressure }\end{array}$ \\
\hline Medium & 0.235 & 0.169 & 1.925 & 0.165 & 1.265 & $0.908-$ \\
\hline High & & & & & & 1.762 \\
\hline Constant & 0.696 & 0.172 & 16.287 & 0.000 & 2.005 & \begin{tabular}{c}
$1.430-$ \\
\hline
\end{tabular} \\
\hline
\end{tabular}

\section{Discussion}

The prevalence of smartphone addiction among the freshmen medical students in this study was $39.7 \%$,which is slightly higher than another study which found that $29.8 \%$ of medical students in China had smartphone addiction ${ }^{[12]}$. Interestingly, in our study, the correlation analysis showed that perceived study pressure was positively correlated with smartphone addiction, whereas gender was not correlated with smartphone addiction. The changes in learning and lifestyle brought about by the COVID-2019 epidemic completely rely on the internet and smart devices, such as tablets, laptops and mobile phones. Unfortunately, this total dependence has proven to be a form of addiction ${ }^{[24]}$. Smartphone is one of the most accessible substances worldwide.Recently, concern was expressed about the increase in smartphone use among students due to the decrease or absence of supervision.To reduce smartphone use, it is sometimes possible to take action against environmental stress factors; however, in the event of epidemics or natural disasters, it is not possible.

During the current COVID-19 pandemic, smartphone is the easiest available substances for managing stress and can lead to the risk of addiction.Such crisis management procedures including isolation, social distancing, continuous confinement, wearing masks, and cancellation of family, social and cultural activities in addition to individual health issues are all potential stressors ${ }^{[25,26]}$. It is suggested that the the negative impact of COVID-19 on mental health beyond health-related fears ${ }^{[27]}$ and can lead to negative psychological effects ${ }^{[28,29]}$. COVID-19 could also have negative impact on mental health through a direct 
effect of the virus in the brain. Therefore, COVID-19 could strengthen the use of addictive substances as strategies to cope with or relieve stress ${ }^{[30,31]}$.Nevertheless, the results of high prevalence indicate that smartphone addiction may be a serious addiction problem, which has a significant impact on the medical students' own lives and studies. The current COVID-19 crisis requires new ways to identify health conditions, while maintaining the principles of reducing human contact and pollution.In this case, a proactive approach to monitoring smartphone use in the student is essential.

This study indicates that professional identity is a positive personal trait and an important protective factor. It can prevent individuals from being affected by perceived stress, thereby reducing their negative emotions and the possibility of developing smartphone addiction.Many studies have pointed out that the professional identity of medical students has a positive impact on their learning motivation,academic success and professional development, and it is also the most important factor influencing the future career choice and professional achievement of medical students ${ }^{[2,33]}$. The professional identity of nursing students could improve their self-confidence, enhance their professional skills ${ }^{[34]}$.Professional Identity could help individuals deal with stress effectively and achieve better adaptation and development. When faced with stress, medical college students with higher levels of professional identity might recover quickly with positive psychological capacity, make greater efforts in the pursuit of success, and have positive expectations and attributes for the results. Therefore, improving the professional identity of medical students can play a role in preventing smartphone addiction.

Abundant studies have shown that poor mental health is positively correlated with smartphone addiction ${ }^{[35]}$.Poor mental health is one of the most important risks leading to smartphone addiction.A study showed that there is a significant positive correlation between depression and smartphone addiction ${ }^{[36]}$.A significant correlation has been shown between perceived vulnerability to disease (PVD) and emotional distress ${ }^{[37]}$. The fear of contagion has been shown as one of the causes of anxiety, depression, and stress in the general population. University student'response to the pandemic deserves further attention. The sudden changes in "University" habits (i.e., poor interaction with teachers and colleagues, difficulty in adapting to online learning), the loss of social networks, and other issues have seriously affected the physical and mental health of University students.A study carried out in China found that about one-third of the respondents have moderate-to-severe anxiety , a high prevalence of sleep disorders, and widespread symptoms of anxiety disorder, especially in young people and health professionals ${ }^{[38,39]}$.Due to the portability, and wide choice of applications on smartphones, poor mental health individuals will make up for the shortcomings in their real social life by using various applications on smartphones. Elhai et al. found that COVID-19 anxiety was related to the severity of depression,anxiety,and problematic smartphone use (PSU) ${ }^{[40]}$.It is increasingly becoming clear that excessive use of smartphones has negative impact on health.

In line with previous studies ${ }^{[41-43]}$, our research found that perceived stress was positively correlated with smartphone addiction among medical freshmen students. The impact of perceived stress on smartphone addiction has begun to gain research support ${ }^{[41,44]}$. As a special group of college students, medical 
students have a high level of stress that could be due to academic burden, frequency of examinations, long academic curriculum ${ }^{[45]}$. Addictive behavior may be a way to reduce strain or relieve negative emotions.Individuals who feel more stress are more likely to engage in smartphone addiction ${ }^{[43]}$. When faced with stress, medical students are more likely to use smartphones to relieve stress ${ }^{[44]}$.Perceived stress could make individuals believe they are in a state of stress, which is considered to be a risk factor for the occurrence and recurrence of many addictions, such as drug abuse ${ }^{[46]}$, internet addiction ${ }^{[47]}$, and so on. The results shown that it is very important for counselors to improve medical college students' Professional Identity in the context of smartphone addiction.In addition, for future clinical practitioners, we must not only pay attention to the symptoms of smartphone addicts themselves, but also their stress and mental health.

Prior literature have shown that prolonged contact with smartphone screens has been reduce sleep time and lower sleep efficiency.The COVID-19 pandemic home quarantine brought about a sudden and different lifestyle, many students watching movies/series most of the time. Overuse of smartphones usually resulted in smartphone addiction.

Some limitations must be admitted. First, this study was conducted in a sample recruited through mobile application and admitted through face-to-face data collection. Therefore, it is possible that some data could be erroneous.Second, this study was cross-sectional and cannot infer causality. Meanwhile, the representative of the sample may limit the general validity of our results, because our participants were from the same university.

In summary, this research reveals the relationship between smartphone addiction and professional identity, mental health, and perceived stress for the first time.Therefore, it is necessary to conduct a largescale longitudinal study to further determine the causal relationship between smartphone addiction, mental health, and professional identity.

\section{Declarations}

\section{Ethics approval and consent to participate}

The design and procedures for this study have been approved by the Ethics Committee of Wannan Medical College, and all participants provide an electronic informed consent form prior to participating in the study. These participants can opt out at any time without providing any reason. All data collection activities for this study were carried out with the informed consent to the participants. All methods are implemented in accordance with the Helsinki Declaration.

\section{Consent for publication}

All authors consent for publication.

\section{Availability of data and materials}


The datasets used and/or analyzed during the current study are available from the corresponding author (H. L or M. Z) on reasonable request.

\section{Competing interests}

All authors declare that no potential conflicts of interest $\$ and consent for publication.

\section{Funding}

This research was funded by MOE (Ministry of Education in China) Project of Humanities and Social Sciences (20YJC190006), The Teaching Quality and Teaching Reform Project of Anhui Provincial Department of Education (2020jyxm2076), School project of the University Student Mental Health Education Research Center of Wannan Medical College (SJD202110), Teaching reform project of Wannan Medical College(2020jyxm58) and the prevention and control science and technology emergency project for COVID-19 of Wuhu (2020rkx1-5).

\section{Authors' contributions}

Huan Liu and Ming Zhang contributed in designed the study and conceived the survey, statistical analysis and wrote the paper. Zhiqing Zhou was involved in the fieldwork. Ergang Zhu played a role in participants' recruitment and data collection within this study. Long Huang and Ming Zhang proposed the research design. All authors have approved the final manuscript.

\section{Acknowledgments}

The authors would like to thank all medical students who completed questionnaires for their actively participation and all the class leaders and counselors of Wannan Medical College for their excellent efforts made this study possible.

\section{References}

1. China Internet Network Information Center. The 44th China Statistical Report on Internet Development. (2019). Available online at: http://www.cac.gov.cn/2019-08/30/c_1124938750.htm (accessed January 7, 2021).

2. Panova T, Carbonell X. Is smartphone addiction really an addiction?. J Behav Addict. 2018;7(2):252259.

3. Guo N, Wang MP, Luk TT, et al. The association of problematic smartphone use with family wellbeing mediated by family communication in Chinese adults: A population-based study. J Behav Addict. 2019;8(3):412-419.

4. Demir YP, Sumer MM. Effects of smartphone overuse on headache, sleep and quality of life in migraine patients. Neurosciences (Riyadh). 2019;24(2):115-121. 
5. Mustafaoglu R, Yasaci Z, Zirek E, et al. The relationship between smartphone addiction and musculoskeletal pain prevalence among young population: a cross-sectional study. Korean J Pain. 2021;34(1):72-81.

6. Kim HJ, Min JY, Min KB, et al. Relationship among family environment, self-control, friendship quality, and adolescents' smartphone addiction in South Korea: Findings from nationwide data. PLoS One. 2018;13(2): e01 90896.

7. Xie $X$, Dong $Y$, Wang J. Sleep quality as a mediator of problematic smartphone use and clinical health symptoms. J Behav Addict. 2018;7(2):466-472.

8. Lee S, Kim HJ, Choi HG, et al. Smartphone Addiction and Interpersonal Competence of Nursing Students. Iran J Public Health. 2018;47(3):342-349.

9. Wang P, Zhao M, Wang $X$, et al. Peer relationship and adolescent smartphone addiction: The mediating role of self-esteem and the moderating role of the need to belong. $J$ Behav Addict. 2017;6(4):708-717.

10. Long J, Liu TQ, Liao YH, et al. Prevalence and correlates of problematic smartphone use in a large random sample of Chinese undergraduates. BMC Psychiatry. 2016;16(1):408.

11. Mao Y, Zhang N, Liu J, et al. A systematic review of depression and anxiety in medical students in China. BMC Med Educ. 2019;19(1):327.

12. Chen B, Liu F, Ding S, et al. Gender differences in factors associated with smartphone addiction: a cross-sectional study among medical college students. BMC Psychiatry. 2017;17(1):341.

13. Karki S, Singh JP, Paudel G, et al. How addicted are newly admitted undergraduate medical students to smartphones?: a cross-sectional study from Chitwan medical college, Nepal. BMC Psychiatry. 2020;20(1):95.

14. Luk TT, Wang MP, Shen C, et al. Short version of the Smartphone Addiction Scale in Chinese adults: Psychometric properties, sociodemographic, and health behavioral correlates. J Behav Addict. 2018; 7 (4):1157-11 65.

15. Yue $H$, Zhang $X$, Sun J, et al. The relationships between negative emotions and latent classes of smartphone addiction. PLoS One. 2021;16(3):e0248555.

16. Li L, Griffiths MD, Mei S, et al. Fear of Missing Out and Smartphone Addiction Mediates the Relationship Between Positive and Negative Affect and Sleep Quality Among Chinese University Students. Front Psychiatry. 2020;11:877.

17. Luo Y, Gong B, Meng R, et al. Validation and application of the Chinese version of the Perceived Stress Questionnaire (C-PSQ) in nursing students. PeerJ. 2018;6:e4503.

18. Sun Y, Luk TT, Wang MP, et al. The reliability and validity of the Chinese Short Warwick-Edinburgh Mental Well-being Scale in the general population of Hong Kong. Qual Life Res. 2019;28(10):28132820.

19. Zhao Y, Zhou Q, Li J, et al. Influence of psychological stress and coping styles in the professional identity of undergraduate nursing students after the outbreak of COVID-19: A cross-sectional study in China [published online ahead of print, 2021 May 7]. Nurs Open. 2021;10.1002/nop2.902. 
20. Wang L, Yang Y, Zhu J, et al. Professional identity and mental health of rural-oriented tuition-waived medical students in Anhui Province, China. BMC Med Educ. 2019;19(1):199.

21. Kwon M, Kim DJ, Cho H, et al. The smartphone addiction scale: Development and validation of a short version for adolescents. PLoS One. 2013;8:e83558.

22. Qin PB. The characteristics and correlation study of college students' specialty identity [Chinese]. Master thesis: Southwest University; 2009.

23. Li T, Zhang $X$, Chen $M$, et al. Psychological distress and its associated risk factors among university students. Rev Assoc Med Bras (1992). 2020;66(4):414-418.

24. Maddux JF, Desmond DP. Addiction or dependence? Addiction. (2000) 95:661-5.

25. Brooks SK, Webster RK, Smith LE, Woodland L, Wessely S, Greenberg N, Rubin GJ. The psychological impact of quarantine and how to reduce it: rapid review of the evidence. Lancet. $2020 \mathrm{Mar}$ 14;395(10227):912-920.

26. Dubey MJ, Ghosh R, Chatterjee S, Biswas P, Chatterjee S, Dubey S. COVID-19 and addiction. Diabetes Metab Syndr. 2020 Sep-Oct;14(5):817-823.

27. Patwardhan P. COVID-19: Risk of increase in smoking rates among England's 6 million smokers and relapse among England's 11 million ex-smokers. BJGP Open. 2020 Jun 23;4(2):bjgpopen20X101067.

28. Wu P, Liu X, Fang Y, et al. Alcohol abuse/dependence symptoms among hospital employees exposed to a SARS outbreak. Alcohol Alcohol. 2008 Nov-Dec;43(6):706-12.

29. Zvolensky MJ, Garey L, Rogers AH, et al. Psychological, addictive, and health behavior implications of the COVID-19 pandemic. Behav Res Ther. 2020 Nov;134:103715.

30. Koob G, Kreek MJ. Stress, dysregulation of drug reward pathways, and the transition to drug dependence. Am J Psychiatry. 2007 Aug;164(8):1149-59.

31. Koob GF, Schulkin J. Addiction and stress: An allostatic view. Neurosci Biobehav Rev. 2019 Nov; 106:245-262.

32. Browne C, Wall P, Batt $S$, et al. Understanding perceptions of nursing professional identity in students entering an Australian undergraduate nursing degree. Nurse Educ Pract. 2018 Sep;32:90-96.

33. Sun L, Gao Y, Yang J, et al. The impact of professional identity on role stress in nursing students: A cross-sectional study. Int J Nurs Stud. 2016 Nov;63:1-8.

34. Miskelly P, Duncan L. 'I'm actually being the grown-up now': leadership, maturity and professional identity development. J Nurs Manag. 2014 Jan;22(1):38-48.

35. Matar Boumosleh J, Jaalouk D. Depression, anxiety, and smartphone addiction in university students- A cross sectional study. PLoS One. 2017 Aug 4;12(8):e0182239.

36. Ghasempour A, Mahmoodi-Aghdam M. The Role of Depression and Attachment Styles in Predicting Students' Addiction to Cell Phones. Addict Health. 2015 Summer-Autumn;7(3-4):192-7.

37. Jungmann SM, Witthöft M. Health anxiety, cyberchondria, and coping in the current COVID-19 pandemic: Which factors are related to coronavirus anxiety? J Anxiety Disord. 2020 Jun;73:102239. 
38. Huang Y, Zhao N. Generalized anxiety disorder, depressive symptoms and sleep quality during COVID-19 outbreak in China: a web-based cross-sectional survey. Psychiatry Res. 2020 Jun;288:112954.

39. Wang C, Pan R, Wan X, et al. Immediate Psychological Responses and Associated Factors during the Initial Stage of the 2019 Coronavirus Disease (COVID-19) Epidemic among the General Population in China. Int J Environ Res Public Health. 2020 Mar 6;17(5):1729.

40. Elhai JD, Yang H, McKay D,et al. COVID-19 anxiety symptoms associated with problematic smartphone use severity in Chinese adults. J Affect Disord. 2020 Sep 1;274:576-582.

41. Chiu SI. The relationship between life stress and smartphone addiction on taiwanese university student: A mediation model of learning self-Efficacy and social self-Efficacy. Computers in Human Behavior, 2014, 34(may):49-57.

42. Kuang-Tsan C , Fu-Yuan H. Study on Relationship Among University Students' Life Stress, Smart Mobile Phone Addiction, and Life Satisfaction. Journal of Adult Development, 2017, 24(2):1-10.

43. Liu Q Q , Zhang D J , Yang X J , et al. Perceived stress and mobile phone addiction in Chinese adolescents: A moderated mediation model. Computers in Human Behavior, 2018, 87(OCT.):247-253.

44. Yang X, Wang P, Hu P. Trait Procrastination and Mobile Phone Addiction Among Chinese College Students: A Moderated Mediation Model of Stress and Gender. Front Psychol. 2020 Dec 1;11:614660.

45. Gazzaz ZJ, Baig M, Al Alhendi BSM, et al. Perceived stress, reasons for and sources of stress among medical students at Rabigh Medical College, King Abdulaziz University, Jeddah, Saudi Arabia. BMC Med Educ. 2018 Feb 23;18(1):29.

46. Tavolacci MP, Ladner J, Grigioni S, et al. Prevalence and association of perceived stress, substance use and behavioral addictions: a cross-sectional study among university students in France, 20092011. BMC Public Health. 2013 Aug 6;13:724.

47. Jun S., Choi E.Academic stress and internet addiction from general strain theory framework. Computers in Human Behavior, 2015, 49:282-287. 